Bull. Austral. Math. Soc.

$46 \mathrm{~B} 20,46 \mathrm{~B} 08$

VoL. 64 (2001) [51-61]

\title{
A 3-SPACE PROBLEM RELATED TO THE FIXED POINT PROPERTY
}

\author{
Helga Fetter and Berta Gamboa de Buen
}

\begin{abstract}
We study some properties which imply weak normal structure and thus the fixed point property. We investigate whether the latter two properties are inherited by spaces obtained by direct sum with a finite dimensional space. We exhibit a space $X$ which satisfies Opial's condition, $X \oplus \mathbb{R}$ does not have weak normal structure but $X \oplus \mathbb{R}$ has the fixed point property.
\end{abstract}

\section{INTRODUCTION}

In recent times several properties implying the fixed point property in Banach spaces have appeared in the literature. The object of this work is to study which of these properties are inherited by spaces obtained by direct sum with a finite dimensional space. In case the latter holds, we shall say that the property is $F D S P$. This is different to the permanence properties under symmetric sums studied by Landes in [4] and [5] and by Sims and Smyth in [7], since besides the sum being symmetric, they consider sums of the space with itself.

The main result is that there exists a space $X$ which satisfies Opial's condition and $X \bigoplus \mathbb{R}$ for a certain family of norms does not have weak normal structure, but $X \bigoplus \mathbb{R}$ still has the fixed point property.

\section{Properties Which are FDSP}

DEFinition 2.1: Let $\mathcal{P}$ be a property of Banach spaces and suppose that each time that a Banach space $Y$ has a finite codimensional subspace $X$ with $\mathcal{P}, Y$ also has $\mathcal{P}$. Then we say that the property is $F D S P$.

We shall show that several of the known properties which are sufficient for weak normal structure and thus for the fixed point property, are FDSP. Next we shall define those properties.

Received 21st August, 2000

Both authors supported partially by Conacyt Grant 32152-E

Copyright Clearance Centre, Inc. Serial-fee code: 0004-9727/01 \$A2.00+0.00. 
Definition 2.2: Let $X$ be a Banach space.

1. $X$ has the fixed point property $(F P P)$ if for every weakly compact subset $K$ of $X$, every non expansive mapping $T: K \rightarrow K$, has a fixed point.

2. $X$ has weak normal structure $(W N S)$ if $X$ does not contain any weakly null sequence $\left\{x_{n}\right\}_{n}$ such that for every $x$ in the closed convex hull of $\left\{x_{n}\right\}_{n}$

$$
\operatorname{diam}\left\{x_{n}\right\}=\lim _{n}\left\|x_{n}-x\right\| \text {. }
$$

3. If there exists $\varepsilon>0$ so that for every weakly null sequence $\left\{x_{n}\right\}_{n} \subset X$ with $\lim _{n}\left\|x_{n}\right\|=1$,

$$
\lim _{n} \sup _{i, j \geqslant n}\left\|x_{i}-x_{j}\right\|>1+\varepsilon,
$$

we say that $X$ satisfies Bynum's condition $(B C)$.

4. If for every weakly null sequence $\left\{x_{n}\right\}_{n} \subset X$ with $\liminf _{n}\left\|x_{n}\right\|>0$ there exists $0<r<1$ such that for every subsequence $\left\{y_{n}\right\}_{n} \subset\left\{x_{n}\right\}_{n}$,

$$
\lim \sup _{n}\left\|y_{n}\right\| \leqslant r \operatorname{diam}\left\{y_{n}\right\}
$$

we say that $X$ has the strong subsequential property $(S S u P)$.

5. If for every weakly null sequence $\left\{x_{n}\right\}_{n} \subset X$ such that $\lim _{n}\left\|x_{n}\right\|=1$ exists,

$$
1<\inf \left\{\operatorname{diam}\left\{y_{n}\right\}:\left\{y_{n}\right\}_{n} \text { is a subsequence of }\left\{x_{n}\right\}_{n}\right\}=A^{*}\left\{x_{n}\right\},
$$

then $X$ has the subsequential property $(S u P)$.

6. If for every weakly null sequence $\left\{x_{n}\right\}_{n} \subset X$

$$
\limsup _{n}\left\|x_{n}\right\|<\lim _{n} \sup _{i, j \geqslant n}\left\|x_{i}-x_{j}\right\|,
$$

then $X$ has the generalised Gossez Lami-Dozo property (GGLD).

Details about FPP and $W N S$ can be found in [2]. The definition of $B C$ is not the original given by Bynum in [1], but an equivalent found in [8]. Properties $S S u P$ and $S u P$ were defined by Sims and Smyth also in [8], and GGLD was defined by Jiménez-Melado in [6].

Theorem 2.3. Properties BC, SSuP, SuP and GGLD are FDSP.

ProOF: We shall only give the proof of the theorem for spaces with codimension one, however the generalisation to spaces of any finite codimension is trivial.

Let $Y$ be a Banach space and $X$ be a codimension one subspace. Then there is $z \in Y$ such that every element of $Y$ is of the form $y=x+\lambda z$ with $x \in X$ and $\lambda \in \mathbb{R}$. Let $\left\{y_{n}\right\}_{n} \subset Y$ be a weakly null sequence. Then

$$
y_{n}=x_{n}+\lambda_{n} z
$$

where $\left\{x_{n}\right\}_{n} \subset X$ is weakly null in $X$ and $\lambda_{n} \underset{n}{\rightarrow} 0$. Also it is easy to see that: 
(a) $\lim \sup _{n}\left\|y_{n}\right\|=\lim \sup _{n}\left\|x_{n}\right\|$ and $\liminf \inf _{n}\left\|y_{n}\right\|=\liminf \operatorname{in}_{n}\left\|x_{n}\right\|$.

(b) $\lim _{n} \sup _{i, j \geqslant n}\left\|y_{i}-y_{j}\right\|=\lim _{n} \sup _{i, j \geqslant n}\left\|x_{i}-x_{j}\right\|$.

(c) $A^{*}\left\{y_{n}\right\}=A^{*}\left\{x_{n}\right\}$.

From (a), (b) and (c) it follows immediately that $B C, S u P$ and $G G L D$ are FDSP. With respect to $S S u P$, suppose that $\liminf \left\|y_{n}\right\|>0$ and that there is $0<r<1$ such that for every subsequence $\left\{u_{n}\right\}_{n}$ of $\left\{x_{n}\right\}_{n}$

$$
\lim \sup _{n}\left\|u_{n}\right\| \leqslant r \operatorname{diam}\left\{u_{n}\right\} \text {. }
$$

Then by (a), $\liminf _{n}\left\|x_{n}\right\|>0$. Now let $\left\{v_{n}\right\}_{n}$ be a subsequence of $\left\{y_{n}\right\}_{n}$ with $v_{n}=u_{n}+\lambda_{m_{n}} z$. Let $\varepsilon>0$ and $N \in \mathbb{N}$ be such that if $i, j>N,\left|\lambda_{m_{i}}-\lambda_{m_{j}}\right|<\varepsilon /\|z\|$. Then

$$
\operatorname{diam}\left\{u_{n}\right\}_{n>N} \leqslant \operatorname{diam}\left\{v_{n}\right\}+\varepsilon
$$

Thus, since $\lim \sup _{n>N}\left\|v_{n}\right\|=\lim \sup _{n}\left\|v_{n}\right\|=\lim \sup _{n}\left\|u_{n}\right\|$, we get

$$
\frac{1}{r} \lim \sup _{n}\left\|v_{n}\right\|=\frac{1}{r} \operatorname{lim\operatorname {sup}_{n}}\left\|u_{n}\right\| \leqslant \operatorname{diam}\left\{u_{n}\right\}_{n>N} \leqslant \operatorname{diam}\left\{v_{n}\right\}+\varepsilon
$$

and thus limsup $\left\|v_{n}\right\| \leqslant r \operatorname{diam}\left\{v_{n}\right\}$. Hence $S S u P$ is also $F D S P$.

The fact that GGLD is FDSP was already noted by Sims and Smyth in [7].

\section{Properties Which are Not FDSP}

There are several properties implying weak normal structure which are found in the literature and which are not $F D S P$. Examples of these are the Gossez Lami-Dozo $(G L D)[3]$, the weakly uniformly Kadec-Klee $(W U K K)$ and the $W U K K^{\prime}[8]$ properties. In fact, if $Y=l_{2} \bigoplus \mathbb{R}$ with the norm $\|(x, \lambda)\|=\max \left(\|x\|_{l_{2}},|\lambda|\right)$, it is easy to see that $l_{2}$ has all the above mentioned properties, but $Y$ does not. However if $X$ has one of the above properties and is of finite codimension in $Y$, then $Y$ has weak normal structure and hence the fixed point property, since all of those properties imply Bynum's condition.

In this section we show that other known conditions related to the fixed point property are not FDSP. We start by recalling the definitions of these properties.

Definition 3.1: Let $X$ be a Banach space.

1. $X$ satisfies Opial's condition $(O C)$ if whenever a sequence $\left\{x_{n}\right\}_{n}$ in $X$ is weakly null, then for $x \neq 0$

$$
\liminf \left\|x_{n}\right\|<\liminf \left\|x_{n}-x\right\| .
$$


2. $X$ satisfies Tingley's condition $(T C)$ if for every weakly null sequence $\left\{x_{n}\right\}_{n}$ in $X$,

$$
\lim \inf _{n}\left\|x_{n}\right\|<\sup _{m}\left(\lim \sup _{n}\left\|x_{m}-x_{n}\right\|\right)
$$

3. $X$ has the weak sum property $(W S P)$ if $X \bigoplus_{l_{1}} \mathbb{R}$ has $W N S$.

Some of these definitions are not the original ones, but are equivalent. The definition of $O C$ can be found in [2]. TC was defined in [9] and WSP in [5]. It is known that

$$
O C \Rightarrow T C \Rightarrow W S P \Rightarrow W N S \Rightarrow F P P \text {. }
$$

DEFINITION 3.2: Let $Y=c$ be the space of converging real sequences $y=\{y(n)\}_{n}$ with the norm $\|\cdot\|_{\alpha}$ for $\alpha \geqslant 0$ :

$$
\|y\|_{\alpha}=\|y\|_{\infty}+\alpha \sum_{i=1}^{\infty} \frac{|y(i)|}{2^{i}}
$$

where $\|y\|_{\infty}=\sup _{n}|y(n)|$. Let $X=\left(c_{0},\|\cdot\|_{\alpha}\right)$ be the subspace of $Y$ consisting of the subsequences convergent to zero.

Recall that a sequence $\left\{y_{n}\right\}_{n}$ in $Y$ is weakly null if and only if $\lim _{n} y_{n}(i)=0$ for every $i \in \mathbb{N}$ and $\lim _{n}\left[\lim _{i} y_{n}(i)\right]=0$.

Theorem 3.3. For $\alpha>0, X=\left(c_{0},\|\cdot\|_{\alpha}\right)$ satisfies Opial's condition.

Proof: Let $\left\{x_{n}\right\}_{n} \subset X$ be a weakly null sequence with $\lim _{n}\left\|x_{n}\right\|_{a}=1$. Then $\lim _{n}\left\|x_{n}\right\|_{\infty}=1, \lim _{n} \sum_{i=1}^{\infty}\left(x_{n}(i)\right) / 2^{i}=0$ and by passing to a subsequence we can assume that there exist $0<i_{1}<i_{2}<\ldots$ such that $\left\|x_{n}\right\|_{\infty}=\left|x_{n}\left(i_{n}\right)\right|$.

Let $x \in X, x \neq 0$; then

$$
\left\|x_{n}-x\right\|_{\alpha} \geqslant\left\|x_{n}-x\right\|_{\infty}+\alpha \sum_{i=1}^{\infty} \frac{|x(i)|}{2^{i}}-\alpha \sum_{i=1}^{\infty} \frac{\left|x_{n}(i)\right|}{2^{i}}
$$

Hence

$$
\lim \inf _{n}\left\|x_{n}-x\right\|_{\alpha} \geqslant \lim \inf _{n}\left\|x_{n}-x\right\|_{\infty}+\alpha \sum_{i=1}^{\infty} \frac{|x(i)|}{2^{i}} .
$$

Now let $\varepsilon>0$ and $J$ be such that for $j \geqslant J,|x(j)|<\varepsilon$ and let $N$ be such that for $n>N, i_{n}>J$. Then for $n>N$

$$
\left\|x_{n}-x\right\|_{\infty} \geqslant\left|x_{n}\left(i_{n}\right)-x\left(i_{n}\right)\right| \geqslant\left|x_{n}\left(i_{n}\right)\right|-\varepsilon
$$

Thus

$$
\lim \inf _{n}\left\|x_{n}-x\right\|_{\infty} \geqslant 1
$$


and

$$
\liminf \left\|x_{n}-x\right\|_{\alpha} \geqslant 1+\alpha \sum_{i=1}^{\infty} \frac{|x(i)|}{2^{i}}>1 .
$$

This finishes the proof.

Jiménez-Melado in [6] mentioned that $\left(c_{0},\|\cdot\|_{\alpha}\right)$ for $\alpha=1$ fails to have property $G G L D$. Thus we obtain the following:

COROLlary 3.4. OC does not imply GGLD.

TheOREM 3.5. For $\alpha \in(0,1], X=\left(c,\|\cdot\|_{\alpha}\right)$ does not have WNS.

Proof: Let $\left\{x_{n}\right\}_{n} \subset X$ be the sequence defined by

$$
x_{n}(i)= \begin{cases}0 & \text { if } i<n \\ 1-\frac{\alpha}{2^{n}} & \text { for } i=n \\ \frac{\alpha}{2^{n}} & \text { for } i>n\end{cases}
$$

Then $\left\{x_{n}\right\}_{n}$ is weakly null,

$$
\left\|x_{n}\right\|_{\alpha}=1-\frac{\alpha}{2^{n}}+\frac{\alpha}{2^{n}}\left(1-\frac{\alpha}{2^{n}}+\sum_{i=n+1}^{\infty} \frac{\alpha}{2^{i}}\right) \underset{n \rightarrow \infty}{\rightarrow} 1
$$

and

$$
\left\|x_{n}-x_{m}\right\|_{\alpha} \underset{n \rightarrow \infty}{\rightarrow} 1
$$

Also, for $n>1$

$$
\sum_{j=1}^{n}\left|x_{j}(i)-x_{n}(i)\right|=\left|\sum_{j=1}^{n}\left(x_{j}(i)-x_{n}(i)\right)\right|= \begin{cases}1-\frac{\alpha}{2} & \text { for } i=1 \\ \left(\sum_{j=1}^{i-1} \frac{\alpha}{2^{j}}\right)+1-\frac{\alpha}{2^{i}} & \text { for } 1<i<n \\ \sum_{j=1}^{n-1}\left(1-\frac{\alpha}{2^{n}}-\frac{\alpha}{2^{j}}\right) & \text { for } i=n \\ \sum_{j=1}^{n-1}\left(\frac{\alpha}{2^{j}}-\frac{\alpha}{2^{n}}\right) & \text { for } i>n .\end{cases}
$$

On the other hand, if $n>3, \sum_{j=1}^{n}\left\|x_{j}-x_{n}\right\|_{\infty}=\sum_{j=1}^{n-1}\left(1-\left(\alpha / 2^{j}\right)\right)$ and

$$
\left\|\sum_{j=1}^{n}\left(x_{j}-x_{n}\right)\right\|_{\infty}=\sum_{j=1}^{n-1}\left(1-\frac{\alpha}{2^{n}}-\frac{\alpha}{2^{j}}\right) .
$$

Hence

$$
\sum_{j=1}^{n}\left\|x_{j}-x_{n}\right\|_{\infty}-\left\|\sum_{j=1}^{n}\left(x_{j}-x_{n}\right)\right\|_{\infty}=\frac{(n-1) \alpha}{2^{n}} \underset{n \rightarrow \infty}{\rightarrow} 0
$$


Thus by (3.4) and (3.5)

$$
\lim _{n \rightarrow \infty}\left(\sum_{j=1}^{n}\left\|x_{j}-x_{n}\right\|_{\alpha}-\left\|\sum_{j=1}^{n}\left(x_{j}-x_{n}\right)\right\|_{\alpha}\right)=0^{\circ}
$$

and by (3.2), (3.3) and (3.6), using a result of Landes found in [4] we get that $X$ does not have $W N S$.

The following result is an immediate consequence of the previous theorem, Theorem 3.3 and (3.1).

Corollary 3.6. Properties $O C, T C, W S P$ and $W N S$ are not FDSP.

Next we shall see that the spaces $\left(c,\|\cdot\|_{\alpha}\right)$ in spite of not having $W N S$ still have FPP. The proof follows along the lines of Lin's proof that spaces with a 1-unconditional basis have FPP (see for example, [2]).

Theorem 3.7. For $\alpha \in[0,1), X=\left(c,\|\cdot\|_{\alpha}\right)$ has the fixed point property.

Proof: Let $K$ be a weakly compact convex set in $X$ and suppose $T: K \rightarrow K$ is a non expansive mapping without a fixed point. We may suppose that $K$ is a minimal $T-$ invariant weakly compact convex set with $\operatorname{diam} K=1$ and that there is a weakly null approximate fixed point sequence $\left\{x_{n}\right\}_{n} \subset K$ for $T$ such that:

(1) $\lim _{n}\left\|x_{n}\right\|_{\alpha}=1$,

(2) $\lim _{n}\left\|x-x_{n}\right\|_{a}=1$ for every $x \in K$,

(3) $\lim _{n}\left\|x_{n}-x_{n+1}\right\|_{\alpha}=1$.

Since $\left\{x_{n}\right\}_{n}$ is weakly null:

(4) $\lim _{n} x_{n}(i)=0$ for every $i \in \mathbb{N}$ and if $k_{n}=\lim _{i} x_{n}(i)$, then $\lim _{n} k_{n}=0$.

Hence we have also, by passing to a subsequence and taking $-K$ if necessary:

(5) $\lim _{n} \alpha \sum_{i=1}^{\infty}\left(\left|x_{n}(i)\right| / 2^{i}\right)=0$.

(6) $\lim _{n}\left\|x_{n}\right\|_{\infty}=1$

(7) There exist $i_{1}<i_{2}<\ldots$ such that $\left\|x_{n}\right\|_{\infty}=x_{n}\left(i_{n}\right)$.

Then, if for $x \in K, \lim _{i} x(i)=k_{x}$, we have $\left|k_{x}\right| \leqslant 1$ and

$$
\left\|x-x_{n}\right\|_{\alpha} \geqslant\left|x\left(i_{n}\right)-x_{n}\left(i_{n}\right)\right|+\alpha \sum_{i=1}^{\infty} \frac{\left|x(i)-x_{n}(i)\right|}{2^{i}}
$$

Thus, by (2), (4) (5), (6) and (7), $1 \geqslant 1-k_{x}+\alpha \sum_{i=1}^{\infty}\left(|x(i)| / 2^{i}\right)$ and

$$
k_{x} \geqslant \alpha \sum_{i=1}^{\infty} \frac{|x(i)|}{2^{i}} \geqslant 0 .
$$


On the other hand, suppose that there exist $r_{1}<r_{2}<\ldots$ such that $x_{n}\left(r_{n}\right)<0$. If $x \in K$, then $1 \geqslant\left\|x-x_{n}\right\|_{\alpha} \geqslant\left|x\left(r_{n}\right)-x_{n}\left(r_{n}\right)\right|+\alpha \sum_{i=1}^{\infty}\left(\left|x(i)-x_{n}(i)\right| / 2^{i}\right)$. Hence $1 \geqslant k_{x}+\limsup \left|x_{n}\left(r_{n}\right)\right|+\alpha \sum_{i=1}^{\infty}\left(|x(i)| / 2^{i}\right)$ and thus

$$
\lim \sup _{n}\left|x_{n}\left(r_{n}\right)\right| \leqslant 1-k_{x}-\alpha \sum_{i=1}^{\infty} \frac{|x(i)|}{2^{i}}
$$

By a standard procedure, using (4), we may extract a subsequence of $\left\{x_{n}\right\}_{n}$ which we shall keep calling $\left\{x_{n}\right\}_{n}$, and construct finite sets $F_{1}, F_{2}, \ldots$ with $\sup F_{i}<\inf F_{i+1}$ and projections $P_{n}: X \rightarrow X$ such that

$$
1 \geqslant\left\|P_{n} x_{n}\right\|_{a}>1-\frac{1}{2^{n}} \text { and }\left\|\left(I-P_{n}\right) x_{n}\right\|_{a}<\frac{1}{2^{n}},
$$

where for $x \in X,\left(P_{n} x\right)(j)= \begin{cases}x(j) & \text { if } j \in F_{n} \\ 0 & \text { otherwise }\end{cases}$

Observe that

$$
P_{n} \circ P_{m}=0 \text { if } n \neq m \text { and }\left\|P_{n}\right\|=\left\|P_{n}+P_{n+1}\right\|=\left\|I-P_{n}\right\|=1 .
$$

and for $x \in X$,

$$
\lim _{n}\left\|P_{n} x\right\|_{\alpha}=k_{x}
$$

Now define for $x \in X$

$$
\left(R_{n} x\right)(j)= \begin{cases}0 & \text { if } j \notin F_{n} \\ x(j)-k_{x} & \text { if } j \in F_{n}\end{cases}
$$

Hence

$$
\begin{gathered}
R_{n} \circ R_{m}=0 \text { if } n \neq m,\left\|R_{n}\right\|=\left\|R_{n}+R_{n+1}\right\|=2, \\
\left\|R_{n} x\right\|_{\alpha}=\max _{i \in F_{n}}\left|x(i)-k_{x}\right|+\alpha \sum_{i \in F_{n}} \frac{\left|x(i)-k_{x}\right|}{2^{i}} \underset{n \rightarrow \infty}{\rightarrow} 0
\end{gathered}
$$

and

$$
\left\|\left(I-R_{n}\right) x\right\|_{\alpha}=\sup \left\{\left|k_{x}\right|,|x(i)|: i \notin F_{n}\right\}+\alpha\left(\sum_{i \notin F_{n}} \frac{|x(i)|}{2^{i}}+\sum_{i \in F_{n}} \frac{\left|k_{x}\right|}{2^{i}}\right) \underset{n \rightarrow \infty}{\rightarrow}\|x\|_{\alpha} .
$$

Thus

$$
\lim _{n}\left\|I-R_{n}\right\|=1
$$


Also

$$
\left\|\left(I-R_{n}\right) x_{n}\right\|_{\alpha}=\left\|x_{n}-P_{n} x_{n}+k_{n} \chi_{F_{n}}\right\|_{\alpha} \underset{n \rightarrow \infty}{\rightarrow} 0
$$

where $\chi_{F}(j)=\left\{\begin{array}{lc}1 & \text { for } j \in F \\ 0 & \text { otherwise }\end{array}\right.$

By (3.7) there are two cases to consider:

I. For every $x \in K, k_{x}=\alpha \sum_{i=1}^{\infty}\left(|x(i)| / 2^{i}\right)$.

II. There exists $u \in K$ with $k_{u}>\alpha \sum_{i=1}^{\infty}\left(|u(i)| / 2^{i}\right)$.

We now translate some of the above into the terminology of ultrapowers. For details, see for example, [2].

Let $\mathcal{U}$ be a free ultrafilter on $\mathbb{N} . \widetilde{K}$ will denote the set

$$
\widetilde{K}=\{K\}_{u}=\left\{\widetilde{u}=\left\{u_{i}\right\}_{u} \in\{X\}_{u}: u_{i} \in K, i \in \mathbb{N}\right\}
$$

where $\{X\}_{u}=\tilde{X}$ is the ultrapower of $X$. If $\left\{S_{i}\right\}$ is an equibounded family of operators $S_{i}: X \rightarrow X$ we define the operator $\widetilde{S}=\left\{S_{i}\right\}_{U}: \widetilde{X} \rightarrow \widetilde{X}$ by

$$
\widetilde{S} \tilde{u}=\left\{S_{i} u_{i}\right\}_{u}
$$

In particular $\widetilde{T}: \widetilde{K} \rightarrow \widetilde{K}$ is the non expansive mapping given by

$$
\widetilde{T} \tilde{u}=\widetilde{T}\left\{u_{i}\right\}_{\mathcal{U}}=\left\{T u_{i}\right\}_{\mathcal{u}}
$$

We identify $K$ with the subset of $\tilde{K}$ represented by the constant sequences $\{x\}_{U}$ with $x \in K$.

Let $\widetilde{P}=\left\{P_{n}\right\}_{U}, \widetilde{Q}=\left\{P_{n+1}\right\}_{U}, \widetilde{R}=\left\{R_{n}\right\}_{U}$ and $\widetilde{S}=\left\{R_{n+1}\right\}_{U}$. Then by (3.10) and

$$
\|\widetilde{P}\|=\|I-\widetilde{P}\|=\|\widetilde{Q}\|=\|I-\tilde{Q}\|=\|\tilde{P}+\widetilde{Q}\|=\|I-\widetilde{R}\|=\|I-\tilde{S}\|=1
$$

and by (3.12)

$$
\|\widetilde{R}\|=\|\widetilde{S}\|=\|\widetilde{R}+\widetilde{S}\|=2 .
$$

Now let $\widetilde{x}=\left\{x_{n}\right\}_{u}, \tilde{y}=\left\{x_{n+1}\right\}_{u}$. Then since $\left\{x_{n}\right\}_{n}$ is an approximate fixed point sequence for $T$,

$$
\tilde{T} \widetilde{x}=\tilde{x} \text { and } \tilde{T} \tilde{y}=\tilde{y}
$$

By (3.9), (3.10), (3.15) and (3.12)

$$
\widetilde{P} \widetilde{x}=\widetilde{x}, \widetilde{Q} \widetilde{y}=\widetilde{y}, \widetilde{P} \widetilde{y}=0 \text { and } \tilde{Q} \widetilde{x}=0
$$


and

$$
\tilde{R} \tilde{x}=\tilde{x}, \tilde{S} \tilde{y}=\tilde{y}, \tilde{R} \tilde{y}=0 \text { and } \tilde{S} \tilde{x}=0 .
$$

Also by (3.11) and (3.13), for $x \in K$

$$
\|\tilde{P} x\|=\|(\tilde{P}+\tilde{Q}) x\|=k_{x} \text { and }\|\tilde{R} x\|=\|(\tilde{R}+\tilde{S}) x\|=0 .
$$

Finally, by (3), (3.9), and from the fact that $\left\|P_{n} x_{n}-P_{n} x_{n+1}\right\|_{\alpha}=\left\|P_{n} x_{n}+P_{n} x_{n+1}\right\|_{\alpha}$, it follows that

$$
\|\widetilde{x}-\tilde{y}\|=\lim _{\mathcal{U}}\left\|P_{n} x_{n}-P_{n+1} x_{n+1}\right\|=\lim _{u}\left\|P_{n} x_{n}+P_{n+1} x_{n+1}\right\|=\|\widetilde{x}+\widetilde{y}\|=1 .
$$

CASE I. In this case for every $x \in K, 0 \leqslant k_{x} \leqslant \alpha /(\alpha+1)$. If this were not true and $k_{x}>\alpha /(\alpha+1)$, then $\|x\|_{\infty}=\|x\|_{\alpha}-k_{x}<1-\alpha /(\alpha+1)=1 /(\alpha+1)$ and thus, since $k_{x}=\alpha \sum_{i=1}^{\infty}\left(|x(i)| / 2^{i}\right)$ this would yield a contradiction. Let

$$
\widetilde{W}=\left\{\widetilde{w} \in \widetilde{K}:\|\widetilde{w}-x\| \leqslant \frac{1}{2} \text { for some } x \in K,\|\widetilde{w}-\widetilde{x}\| \leqslant \frac{1}{2},\|\widetilde{w}-\widetilde{y}\| \leqslant \frac{1}{2}\right\} .
$$

Clearly $\widetilde{W}$ is a closed and convex subset of $\widetilde{K}$ and by (3.22) $\widetilde{z}=(\widetilde{x}+\widetilde{y}) / 2 \in \widetilde{W}$ (taking $x=0 \in K)$. Also by (3.18) and since $T$ is non expansive,

$$
\|\tilde{T} \tilde{w}-T x\| \leqslant\|\tilde{w}-x\|,\|\tilde{T} \tilde{w}-\tilde{x}\| \leqslant\|\tilde{w}-\tilde{x}\| \text { and }\|\tilde{T} \tilde{w}-\tilde{y}\| \leqslant\|\tilde{w}-\tilde{y}\|,
$$

showing that $\widetilde{W}$ is $\widetilde{T}$ invariant.

Now, if $\widetilde{w} \in \widetilde{W}$, then by (3.19), (3.16) and (3.21) we obtain, since $\alpha<1$,

$$
\begin{aligned}
\|\widetilde{w}\|= & \frac{1}{2}\|(\tilde{P}+\widetilde{Q}) \widetilde{w}+(I-\widetilde{P}) \widetilde{w}+(I-\widetilde{Q}) \widetilde{w}\| \\
\leqslant & \frac{1}{2}(\|(\widetilde{P}+\widetilde{Q})(\tilde{w}-x)\|+\|(\widetilde{P}+\widetilde{Q}) x\| \\
& +\|(I-\widetilde{P})(\widetilde{w}-\widetilde{x})\|+\|(I-\widetilde{Q})(\widetilde{w}-\widetilde{y})\|) \\
\leqslant & \frac{1}{2}\left(\frac{1}{2}+k_{x}+\frac{1}{2}+\frac{1}{2}\right) \leqslant \frac{3}{4}+\frac{\alpha}{2(\alpha+1)}<1,
\end{aligned}
$$

but by Karlovitz's lemma

$$
\sup _{\widetilde{w} \in \widetilde{W}}\|\widetilde{W}\|=1
$$

CASE II. There exists $u \in K$ with $k=k_{u}=\lim _{i}|u(i)|>\alpha \sum_{i=1}^{\infty}\left(|u(i)| / 2^{i}\right)$.

In this case we shall show that there is $0<b<1 / 2$ such that the closed convex set $\widetilde{W}$ given by

$$
\widetilde{W}=\left\{\widetilde{w} \in \widetilde{K}:\|\widetilde{w}-x\| \leqslant \frac{1}{2}-b \text { for some } x \in K,\|\tilde{w}-\tilde{x}\| \leqslant \frac{1}{2},\|\widetilde{w}-\tilde{y}\| \leqslant \frac{1}{2}\right\}
$$


is not empty. Then, as above $\widetilde{W}$ is $\widetilde{T}$ invariant and if $\widetilde{w} \in \widetilde{W}$, by $(3.21),(3.20),(3.17)$ and (3.16).

$$
\begin{aligned}
\|\tilde{w}\| & \leqslant \frac{1}{2}(\|(\widetilde{R}+\widetilde{S}) \tilde{w}\|+\|(I-\widetilde{R}) \tilde{w}\|+\|(I-\tilde{S}) \tilde{w}\|) \\
& =\frac{1}{2}(\|(\widetilde{R}+\widetilde{S})(\widetilde{w}-x)\|+\|(I-\tilde{R})(\widetilde{w}-\tilde{x})\|+\|(I-\tilde{S})(\widetilde{w}-\tilde{y})\|) \\
& \leqslant \frac{1}{2}\left(2\left(\frac{1}{2}-b\right)+\frac{1}{2}+\frac{1}{2}\right)=1-b,
\end{aligned}
$$

and this again contradicts Karlovitz's lemma.

We shall prove now that $(\widetilde{x}+\widetilde{y}) / 2 \in \widetilde{W}$ by exhibiting $x \in K$ and $b<1 / 2$ satisfying $\|(1 / 2)(\tilde{x}+\tilde{y})-x\| \leqslant(1 / 2)-b$. To this end let $u \in K$ as above and $0<\lambda<1$. Since $K$ is convex and $0 \in K, \lambda u \in K$. Now we shall estimate $\left\|\left(P_{n} x_{n}+P_{n+1} x_{n+1}\right) / 2-\lambda u\right\|_{\alpha}$.

$$
\left|\frac{1}{2}\left(P_{n} x_{n}+P_{n+1} x_{n+1}\right)(i)-\lambda u(i)\right|= \begin{cases}\lambda|u(i)| & \text { if } i \notin F_{n} \cup F_{n+1} \\ \left|\frac{x_{r}(i)}{2}-\lambda u(i)\right| & \text { if } r \in\{n, n+1\} \text { and } i \in F_{r} .\end{cases}
$$

Let $0<\varepsilon<k-\alpha \sum_{j=1}^{\infty}|u(j)| / 2^{j}$ and suppose that $n$ is sufficiently large that $u(i)>0$, $|u(i)-k|<\varepsilon$ for $i \in F_{r}$ and if $x_{r}(i)<0$, then by (3.8)

$$
\left|x_{r}(i)\right|<1-k-\alpha \sum_{j=1}^{\infty} \frac{|u(j)|}{2^{j}}+\varepsilon
$$

But

$$
\begin{aligned}
& \mid \begin{array}{ll}
\frac{x_{r}(i)}{2}-\lambda u(i) \mid & \text { if } \lambda u(i)<\frac{x_{r}(i)}{2} \\
\frac{x_{r}(i)}{2}-\lambda u(i)<\frac{1}{2}-\lambda(k-\varepsilon) & \text { if } 0 \leqslant \frac{x_{r}(i)}{2} \leqslant \lambda u(i) \\
0 \leqslant \lambda u(i)-\frac{x_{r}(i)}{2} \leqslant \lambda u(i) \leqslant \lambda(k+\varepsilon) & \text { if } x_{r}(i)<0 . \\
\lambda u(i)+\frac{\left|x_{r}(i)\right|}{2} \leqslant \lambda(k+\varepsilon)+\frac{1}{2}\left(1-k-\alpha \sum_{j=1}^{\infty}\left|\frac{u(j)}{2^{j}}\right|+\varepsilon\right)
\end{array}
\end{aligned}
$$

Let $\lambda$ be sufficiently small that $\lambda|u(i)|<(1 / 2)-\lambda k$ for every $i$ and

$$
2 \lambda k<\frac{k+\alpha \sum_{j=1}^{\infty}\left|u(j) / 2^{j}\right|-\varepsilon}{2} .
$$

Then

$$
\left\|\frac{1}{2}\left(P_{n} x_{n}+P_{n+1} x_{n+1}\right)-\lambda u\right\|_{\alpha} \leqslant\left(\frac{1}{2}-\lambda k+\lambda \varepsilon\right)+\lambda \alpha \sum_{j=1}^{\infty}\left|\frac{u(j)}{2^{j}}\right|+\alpha \sum_{j \in F_{n} \cup F_{n+1}}\left|\frac{x_{r}(j)}{2^{j}}\right|
$$


and finally

$$
\lim _{n}\left\|\frac{1}{2}\left(P_{n} x_{n}+P_{n+1} x_{n+1}\right)-\lambda u\right\|_{\alpha} \leqslant \frac{1}{2}-\lambda k+\lambda \varepsilon+\lambda \alpha \sum_{j=1}^{\infty}\left|\frac{u(j)}{2^{j}}\right|<\frac{1}{2} .
$$

Therefore, if $x=\lambda u,\|(\widetilde{x}+\widetilde{y}) / 2-x\|=(1 / 2)-b$ for some $b>0$ and this concludes the proof.

We do not know of an example of a space having $F P P$ and codimension one in another space without $F P P$. We also do not know if the previous theorem is still valid for $\alpha=1$.

\section{REFERENCES}

[1] W.L. Bynum, 'Normal structure coefficients for Banach spaces', Pacific J. Math. 86 (1980), 427-436.

[2] K. Goebel and W.A. Kirk, Topics in metric fixed point theory, Cambridge Studies in Advanced Mathematics 28 (Cambridge University Press, Cambridge, 1990).

[3] J.P. Gossez and E. Lami Dozo, 'Structure normale et base de Schauder', Acad. Roy. Belg. Bull. Cl. Sci. 55 (1969), 673-681.

[4] T. Landes, 'Permanence properties of normal structure', Pacific J. Math. 110 (1984), 125-143.

[5] T. Landes, 'Normal structure and the sum-property.', Pacific J. Math. 123 (1986), 127-147.

[6] A. Jiménez-Melado, 'Stability of weak normal structure in James quasi reflexive space', Bull Austral. Math. Soc. 46 (1992), 367-372.

[7] B. Sims and M. Smyth, 'On non-uniform conditions giving weak normal structure', Quaestiones Math. 18 (1995), 9-19.

[8] B. Sims and M. Smyth, 'On some Banach space properties sufficient for weak normal structure and their permanence properties', Trans. Amer. Math. Soc. 351 (1999), 497-513.

[9] D. Tingley, 'The normal structure of James quasi reflexive space', Bull. Austral. Math. Soc. 42 (1990), 95-100.

CIMAT

Apdo. Postal 402

36000 Guanajuato

Gto. México 